\title{
Laser-driven ion accelerators for tumor therapy revisited
}

\author{
Ute Linz ${ }^{1, *}$ and Jose Alonso ${ }^{2, \dagger}$ \\ ${ }^{1}$ Forschungszentrum Jülich, D-52425 Jülich, Germany \\ ${ }^{2}$ Lawrence Berkeley National Laboratory, Berkeley, California 94720, USA
}

(Received 2 August 2016; published 29 December 2016)

\begin{abstract}
Ten years ago, the authors of this report published a first paper on the technical challenges that laser accelerators need to overcome before they could be applied to tumor therapy. Among the major issues were the maximum energy of the accelerated ions and their intensity, control and reproducibility of the laserpulse output, quality assurance and patient safety. These issues remain today. While theoretical progress has been made for designing transport systems, for tailoring the plumes of laser-generated protons, and for suitable dose delivery, today's best lasers are far from reaching performance levels, in both proton energy and intensity to seriously consider clinical ion beam therapy (IBT) application. This report details these points and substantiates that laser-based IBT is neither superior to IBT with conventional particle accelerators nor ready to replace it.
\end{abstract}

DOI: 10.1103/PhysRevAccelBeams.19.124802

\section{INTRODUCTION}

"What will it take for laser driven proton accelerators to be applied to tumor therapy?" was the title of a first article by Linz and Alonso on the challenges for laser-driven proton accelerators in 2007 [1]. In the intervening ten years considerable theoretical progress has been made on handling of the less-than-ideal beams of protons that might be produced with lasers, but progress on the necessary lasers has been a lot slower than anticipated. Proton energies and intensities are still not close to the levels suitable for therapy applications, even with the most powerful lasers. The bold predictions for rapid advances towards clinically viable laser-based proton accelerator systems [2-6] have proven premature. Meanwhile, proton therapy facilities based on conventional accelerators continue to expand, with great strides being made in size and cost reduction $[7,8]$.

In the past ten years the total number of patients treated with ion beam therapy (IBT) from conventional accelerators has more than tripled (155000 vs 48000). Roughly 17000 patients are currently treated per year, approximately twice as many as in 2007. The number of IBT facilities has more than doubled from 27 to 62. In 2016, more than 30 additional IBT centers are under construction [9]. As now several new facilities for ions heavier than protons have opened, the ratio of proton to carbon ion therapy has shifted from 10:1 to $6: 1$ (Fig. 1).

\footnotetext{
*Corresponding author. u.linz@fz-juelich.de

†RAlonso@LBL.gov
}

Published by the American Physical Society under the terms of the Creative Commons Attribution 4.0 International license. Further distribution of this work must maintain attribution to the author(s) and the published article's title, journal citation, and DOI.
Perhaps more importantly, there are today at least seven commercial vendors actively marketing proton therapy systems. Ion Beam Applications (IBA), Varian, Mevion, Hitachi, Sumitomo, Mitsubishi, and Protom are all building and installing turn-key systems based on different types of cyclotrons and synchrotrons. All these systems are delivered with pencil-beam scanning systems capable of exquisite dose delivery that easily rival and surpass the best plans available from intensity-modulated radiation therapy (IMRT) and other advanced x-ray modalities (for review cf. [10,11]). These systems have superb reliability records, typically guaranteed at $95 \%$ up-time, and often the time between handover to the customer and beginning of patient treatments is a matter of only a few weeks. These vendors are also actively continuing research to address the barriers to further market penetration: size and cost of the facilities. IBA, Hitachi, Sumitomo and particularly Mevion are among the vendors offering single-room options, with footprints not that much larger than that of a conventional $\mathrm{x}$-ray linac installation. The Mevion system in fact, has a compact, superconducting $9 \mathrm{~T}$ synchrocyclotron mounted directly on the patient gantry. It has no beam lines with bending or focusing magnets between accelerator and patient. These developments are not leveling off yet either, and it is expected that further reductions in size and cost will be seen in the marketplace over the coming years.

Conventional accelerator-based proton therapy has moved from the research laboratory solidly into the commercial sector, where success is based on demonstrated performance and a superior competitive position.

Thus, the task for the laser community becomes even more difficult. The commercial world is rapidly maturing its development of conventional accelerator-based proton therapy systems, and so has a large headstart. This makes the goal of producing a commercially viable, marketable 


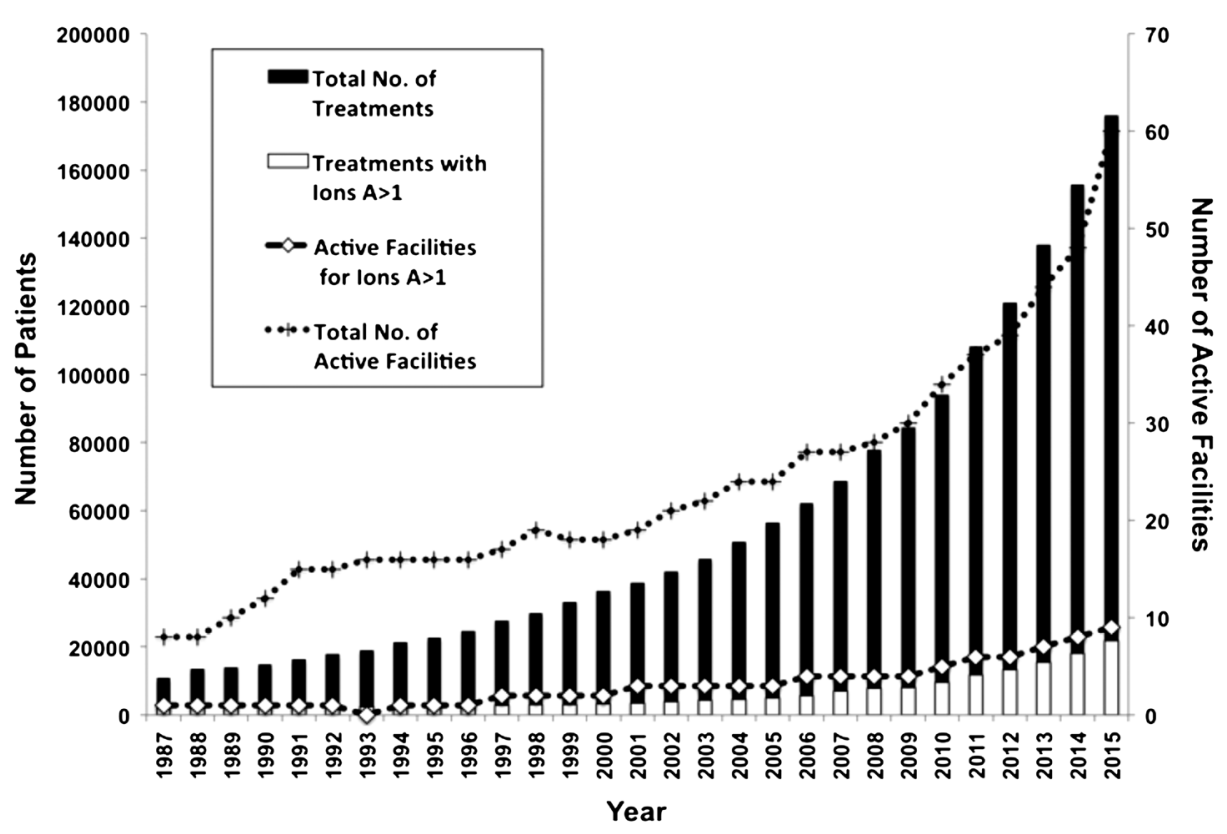

FIG. 1. Number of active ion beam therapy facilities and patients treated. Ions A $>1$ designate ions heavier than protons (data from PTCOG statistics [9]).

laser-based proton therapy facility that can be considered competitive a truly daunting one.

\section{BEAM ENERGY}

In 2000, experiments at the large research laser NOVA (Lawrence Livermore National Laboratory) led to the acceleration of protons up to $58 \mathrm{MeV}[12]$. As of today, this maximum has only marginally been increased to $67 \mathrm{MeV}$ using single shots onto flattop cone targets at the TRIDENT laser of the Los Alamos National Laboratory [13]. TRIDENT is-like NOVA-another high-power, high-energy laser with a repetition rate in the millihertz range. It is hence not relevant for routine clinical usage.

A more recent experimental series at TRIDENT purportedly produced $160 \mathrm{MeV}$ protons. Even though generated at a giant model laser and showing a proton flux at the high-energy tail more than 3 orders of magnitude lower than the flux at $10 \mathrm{MeV}$, it would be a new record. It is, therefore, very surprising that this milestone result hides as a preprint in [14] and has not yet been published in a peer-reviewed journal.

The same Los Alamos group accelerated carbon ions and described an energy leap to approximately $60 \mathrm{MeV}$ per nucleon [6]. This is about 10 times the energy previously reported, but only about $15 \%$ of what is needed for IBT. The authors attribute their "breakthrough" to a "paradigm shift" in the laser-target interaction [14]. Rather than relying on the inefficient surface acceleration in the target normal sheath acceleration (TNSA) mode, they propose to have reached a regime of relativistic target transparency, where an overdense target is rendered transparent. This acceleration mechanism is called break-out-afterburner
(BOA). It requires laser intensities in the $10^{20} \mathrm{~W} / \mathrm{cm}^{2}$ range and long pulses $\geq 500 \mathrm{fs}$, conditions that fit the TRIDENT laser perfectly.

According to Borghesi et al. [15], the carbon ion acceleration has recently been pushed a little further to $80 \mathrm{MeV} / \mathrm{u}$ for $\mathrm{C}^{6+}$. Higher acceleration results are predicted for a third acceleration regime called radiation pressure acceleration (RPA). However, with laser intensities of $>10^{22} \mathrm{~W} / \mathrm{cm}^{2}$ and pulses of $<100 \mathrm{fs}$, this and other acceleration regimes for high-intensity lasers have only been simulated in computer models. Experimentally, it has not been possible to verify them, not even with lasers of the TRIDENT caliber $[6,16]$.

Several upscaling theories exist. Fuchs et al. [17], for example, predicted the proton energy to increase with the square root of the laser power. An increase of the proton energy from 50 to $200 \mathrm{MeV}$ would hence require a 16-fold laser power step-up. Experimental work, however, seems to yield a shallower increase of the ion energy so far $[13,18,19]$. For intensities beyond $5 \times 10^{21} \mathrm{~W} / \mathrm{cm}^{2}$ the TNSA mechanism should be on the wane and replaced by the RPA mode [20]. Whether this will yield the extra push to higher energies needs to be seen.

In 2006, it was projected that compact petawatt lasers would replace conventional cyclotrons for proton therapy by now [21]. However, this has not been the case. Even though there is a number of petawatt lasers operating, none of them is of "tabletop" size and none is able to generate protons of the desired energy at the required flux for cancer therapy.

Maximum energies of around $60 \mathrm{MeV}$ have been established. If one might work from this base and improve 
the spectrum and intensity through laser power and target enhancements, would this be a useful beam for proton therapy?

Uveal melanoma, a tumor of the eye, is frequently mentioned as a potential indication for laser-driven proton therapy [22]. This tumor can indeed be irradiated with proton beams in the $60 \mathrm{MeV}$ range. But is it a meaningful model case for laser accelerators? Could this be a niche where laser-produced protons could be shown to be competitive?

Uveal melanoma is a very rare tumor, about as frequent as male breast cancer (1-8 per million people). Other than protons, there are several alternative treatments for this tumor that are successful under specific conditions, notably, radioactive plaques, and also cryotherapy, photocoagulation, and enucleation (for review cf. for example [23]). One proton facility can easily treat all the cases of a country the size of Germany ( 80 million people). Proton facilities with 60-MeV cyclotrons such as Clatterbridge, UK, University of California, San Francisco, USA, or Catania, Italy do at best treat about 100 patients per year and are certainly not used at full capacity [9].

Are there other tumors for a proton range of less than $8 \mathrm{~cm}$ or $100 \mathrm{MeV}$ ? Not really. Statistics with 3200 patients treated at NIRS, Chiba, Japan, revealed that over a period of 12 years less than $1 \%$ of the patients received ions for such superficial targets [24]. It is, therefore, not wise to build a technological business for such a treatment niche, which can already be served by established methods.

\section{BEAM INTENSITY}

Beam intensity remains a severe challenge for potential laser accelerators. A dose rate of $10^{10}$ protons per second or 2 nanoamperes is required at the patient to achieve a standard fraction of 2 Grays within 2 minutes. Tailoring a beam to meet the specific requirements for treating a tumor invariably involves selecting the ions of the right energy that can be steered to the appropriate element of the target volume. Because of the nature of protons from a laser source, this selection involves collimation, trimming or analysis capabilities in the space between production and patient. It cannot be repeated often enough that any losses between the accelerator (whether laser foil or cyclotron) and the patient have to be compensated for by an accordingly higher ion count from the source of accelerated protons.

One of the highest energies achieved for laser-driven ion acceleration were protons of $67 \mathrm{MeV}$ by Gaillard et al. [13]. But only $7 \times 10^{6}$ protons were in the cutoff energy fraction of a single shot. Even under the model conditions of this experiment with a repetition rate in the millihertz range, the authors commented that the laser alignment was not stable and focusing the laser onto the structured target was difficult to control.
So far, there are again only predictions that novel curved targets, a 10-100 Hz laser and a solenoid collector would yield the $10^{8}$ to $6 \times 10^{8}$ protons per shot required for depth scanning [25-27].

In 2004, Ledingham et al. [28] stated that a practical application of the available lower energy $(40 \mathrm{MeV})$ proton beam would be for isotope production. More recent work [29] acknowledges that isotope yield from dedicated PET cyclotrons is hundreds of times more than from lasers, however, that single-dose amounts are obtainable from lasers. It is difficult to understand how this could compete with the highly efficient, compact and very cost-competitive cyclotrons dedicated to producing PET isotopes. If microfluidic tracer techniques require lower amounts of activity, the cyclotron can be run for a few seconds (instead of one hour required for the laser) to produce the activity required, approaching a true "on-demand" system with minimum wait time for labeled pharmaceuticals.

Summarizing, beam intensities from laser sources are still not sufficient to be a serious challenge to any medical application of conventional accelerators.

\section{ENERGY SPREAD AND DOSE CONFORMATION}

While typical laser-accelerated ion spectra show close to $100 \%$ energy modulation with exponentially decreasing intensity, proton spectra with an energy spread of approximately $10 \%$ containing about $10^{9}$ protons have been reported using an acceleration mode called confined TNSA. The reproducibility was specified as $80 \%$. Unfortunately, peak energies were not higher than $3 \mathrm{MeV}$ [18]. For high laser power densities $\left(\geq 5 \times 10^{21} \mathrm{~W} / \mathrm{cm}^{2}\right)$ where BOA and RPA regimes are to predominate, an energy spread of approximately $4 \%$ is predicted [30]. Other theoretical scaling models promise at best a bandwidth of $1 \%[31,32]$.

Until these fine energy widths are demonstrated, though, any concepts for clinical treatments must use the best available "highly peaked" [33] or "quasimonoenergetic" [18,34] spectra. Schell and Wilkens [35] have developed sample plans for head and neck tumors that yield dosevolume histograms comparable to plans for spot scanning with conventionally generated ion beams. However, to achieve this the raw plume of protons must be run through a magnetic energy-selection and bandwidth acceptance device, and a very sophisticated multileaf collimation system. To avoid a totally unacceptable loss of particles in this selection system, the average energy (between 70 and $250 \mathrm{MeV}$, i.e., energies not yet seen from laser-driven systems) and spectrum (approximately 10\% to 30\% FWHM) of the plume should match as closely as possible the settings of the selection channel, indicating a degree of control over the laser-target interaction that is not yet possible. In addition, a base rate of at least $10^{8}$ protons per pulse, at a $10 \mathrm{~Hz}$ rate must be available to not lengthen the 
treatment time to unacceptable limits. It should be noted, though, that this basic flux is still only about $10 \%$ of the rate for proton beams from cyclotrons or synchrotrons. These plans demonstrate that if the specified proton intensity, mean energy, and energy widths can be flexibly and reliably produced with a laser pulse, it will be possible to generate suitable treatment plans. While these studies are interesting exercises, the basic premises for the beam parameters are not achievable today.

\section{DOSE ACCURACY AND DOSIMETRY TECHNIQUES}

The calculated dose has to be applied with not more than five percent deviation. This requirement is easily met by ion beams from conventional accelerators. However, because of the extremely peaked time structure of beams produced by lasers, this is a major challenge which seems today more recognized than before [22].

A European consortium plans to develop a prototype beam line for radiobiology and dosimetry studies called ELIMED [36]. Development of a new Thomson spectrometer and dosimetric detectors are primary goals, as the special time structure of a laser-driven ion beam asks for ultrafast beam diagnostics and dose detection. The ELI beam line project envisages the development of 1-10 petawatt class lasers. As this is a long-term goal, the various diagnostic elements will first be tested at currently operative lasers, such as the ASTERIX laser in Prague or TARANIS at Queens University, Belfast [37]. Both are TW lasers with repetition rates of one shot per 20 and 10 minutes, respectively [38,39], which means again, very early prototypes of a laser-driven proton accelerator. The final implementation phase foresees a "high repetition-rate" laser [40] with a beam transport line for protons of $100 \mathrm{MeV}$ maximum. However, as of now there is no time schedule provided to reach this still modest energy goal.

Some researchers consider the properties of lasergenerated ion beams as "certainly superior" to those of conventional accelerators and provide as explanation the "vicinage effect," an increased stopping power due to cluster formation of the incident ions [33]. While protons are certainly closely located in space and time at their production site in the target, their angular and energy spread broadens the distribution to where no two protons are close to one another in the treatment volume. The ELIMED beam line would be an ideal place to look for such effects, though, it is doubtful any will be found.

\section{ISOCENTRIC GANTRY}

For optimum treatment results, irradiation with ions requires the possibility to direct the beam from any angle to the target. A flexible robotic patient couch and a rotating beam line, a so-called (isocentric) gantry, provide this option. All commercially available proton therapy facilities offer a gantry option. Early proton gantries were large, typically 13 meters in diameter. This size was driven by the use of the double-scattering beam delivery system which required the entire field-forming system using scattering foils, distal-edge shaping compensators and collimators (referred to as the "nozzle"), to be located after the last bending magnet. However, the now almost universal adoption of pencil beam scanning allows proton gantries to be substantially reduced in size, by placing the scanning magnets before the last bend, thus requiring only the dosimetry package to be located between this magnet and the patient. IBA now offers a compact gantry that is only 7.5 meters in diameter.

Using superconducting rather than normal-conducting magnets provides further means to reduce the gantry size. ProNova is developing superconducting gantries with diameters as low as 6 meters [41]. The benefit of superconducting gantries is even more pronounced with carbon or heavier ion beams. The Heidelberg gantry, with its mass of 670 tons, is continuously singled out as the poster child of the massiveness of IBT facilities. Though large, it does accomplish its aim of isocentric delivery of heavier ions. It was not intended as an example of miniaturization. A big step in size and weight reduction has been taken by the National Institute of Radiological Sciences (NIRS) in Chiba, Japan, which has built a superconducting gantry for carbon ions that is almost the size of a conventional proton gantry [42]. Final tests of it are under way and clinical operation is to start soon.

One of the advertised benefits of protons from a laser source is that an all-optical gantry, using mirrors instead of bending magnets, can provide a substantially more compact and inexpensive means of isocentric beam delivery $[2,3,5]$. As mentioned above, though, the plume of particles from the target must be carefully tailored to meet the requirements of the treatment, i.e., the equivalent of today's gantry nozzle must be provided for. In essentially all of the studies reported, this will contain the energy selection system with magnets and adjustable slits, a sophisticated and fast multileaf collimator to define the specific spot being irradiated, and the dosimetry/monitoring instrumentation package. Because of the rigidity of the high-energy protons, magnetic analysis systems are not small. The most compact calls for an 8.7 tesla solenoid which is still some $70 \mathrm{~cm}$ long [27]. An accordingly designed nozzle will probably be at least two meters in length. Adding vacuum chambers, target changing mechanisms, and the mirrors suitably protected from the target blasts, the radius of the gantry will probably still be over four meters or about the same size as a current compact proton gantry. There would be some savings in that the total magnet weight would be less. However, on the whole, the gantry will not look much different from today's proton gantries. 


\section{RADIATION PROTECTION, ENVIRONMENTAL CONSIDERATIONS}

A substantial amount of the cost for a conventional proton therapy facility lies in the concrete vault in which the beam delivery and patient handling systems are located.

As was explained in the previous section, the gantry diameter of a laser-based proton therapy system will probably not be that different from today's proton gantries. The lateral dimensions of the vault will be the same. The length of the vault could be a bit smaller, however, only about half the size of the vault is used by the gantry. The infrastructure required for the patient accounts for the remainder: complex robotic positioner, positionverification (which more and more includes an in-room CT scanner), control station and storage of material for technicians, and the entrance maze. So, reduction of the gantry length by half has only a small impact on the overall vault size. The thickness of the concrete shielding must be the same, as these are calculated on the beam necessary to deliver the prescribed dose to the patient.

However, other shielding and environmental considerations must be addressed with the laser-generating system. With the target located on the all-optical gantry, and near the patient, shielding must be provided from the $\mathrm{x}$ rays, electrons and neutrons produced in the target, over and above the protons used for the treatment.

In computer simulations, Fan et al. [43] concluded that a two-layer shielding of 10-12 $\mathrm{cm}$ polyethylene and $4 \mathrm{~cm}$ lead would be sufficient for $300 \mathrm{MeV}$ protons and $270 \mathrm{MeV}$ electrons generated at a laser intensity of $2 \times 10^{21} \mathrm{~W} / \mathrm{cm}^{2}$. It is not clear if this simulation calculated shielding for the neutrons produced. This localized shielding must be placed close to the target, and must be designed to tightly enclose the sources of this radiation, taking into account the entry path for the laser light, the targetchanging mechanism and the exit path of the protons.

Another factor that has not been considered so far is sound-proofing. A pulsed $9 \mathrm{~T}$ solenoid, mechanical target changing, and other sources of noise might be an environmental issue that needs to be addressed.

\section{COST}

Cost and size have been the major arguments for the followers and supporters of laser-driven accelerators for IBT. Some consider laser-driven accelerators even a necessity because costs of conventional systems are "prohibitive for many developing countries" [22].

Probably more than the price tag, the size of the equipment has been an understandable reason why tertiary care or community hospitals have hesitated to invest in IBT. However, contrary to the pessimistic predictions of members of the laser acceleration community, compact, singleroom proton therapy units driven by superconducting synchrocyclotrons have become a reality (Fig. 2), and
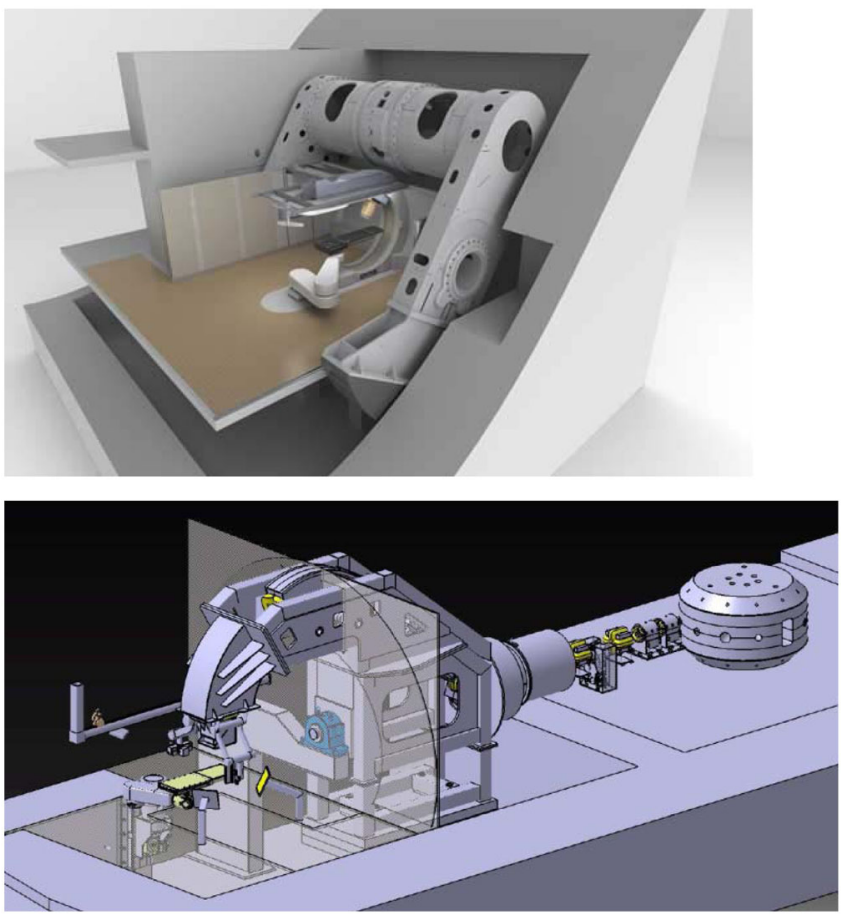

FIG. 2. Two examples of commercial single-room proton therapy facilities. Top: Mevion S250 ${ }^{\mathrm{TM}}$, Mevion, Littleton, MA, USA. Building volume approximately $14 \times 14 \times 14 \mathrm{~m}^{3}$ (data from [7]). Bottom: ProteusOne ${ }^{\mathrm{TM}}$ Ion Beam Applications, Louvain-la-Neuve, Belgium. Building volume approximately $13 \times 14 \times 27 \mathrm{~m}^{3}$ (data from [44]).

entry costs for these single-room facilities have dropped significantly. At least four companies-Mevion, IBA, Hitachi, and Varian — offer gantry-equipped treatment units that provide pencil beam scanning and easily fit into the infrastructure of hospitals and even private clinics (cf. for example Ackerman Cancer Center, Jacksonville, USA). The footprint of the inner room is in the range of $70 \mathrm{~m}^{2}$. For Mevion's S250 system, the outer dimensions including shielding walls are less than $200 \mathrm{~m}^{2}$ and about $260 \mathrm{~m}^{2}$ or the size of a tennis court for Varian's single-room proton therapy solution ProBeam ${ }^{\circledR}$ Compact. At a price of less than 30 million US Dollars (USD) for such a facility including long-term service and maintenance agreement, it is quite difficult to argue that a laser-based therapy center will be much cheaper. In addition, competitive forces are driving these prices even lower.

Unrealistic estimates saw the cost for laser accelerators between 5 and 10 million USD [4]. Roughly 20 million USD were estimated for a "dedicated test facility" with a startup laser of moderate energy "to establish the necessary foundation" [22]. In the light of existing, compact, and robust ion beam facilities that currently treat $17000+$ patients per year, such a preliminary orientation phase seems obsolete. What counts are large numbers of effectively treated patients. 


\section{UNREALISTIC EXPECTATIONS}

Progress towards laser-driven proton therapy has been slow, even if some enthusiasts consider laser-driven ion beams as being in a "phase of maturing technologies" [33] and praise the superiority of laser-based proton beams for therapy, with optimistic claims and projections.

In 2006, for example, a medical physicist of the Fox Chase Cancer Center in Philadelphia, USA, argued that conventional cyclotrons for proton radiotherapy would become obsolete and replaced by far less expensive compact laser systems within a decade [21]. At about the same time, researchers at the Centre national de la recherche scientifique (CNRS) in France announced they would have a first prototype of a medical laser accelerator operational within four years [45]. In 2011, the petawatt laser system for therapy was "envisioned to be built in the near future" [46] and in 2012, the project was updated for another three to five years "at which point therapeutic deployment" should take place [47].

In 2008, the Photo-Medical Research Center near Kyoto, Japan, set up a project to develop a compact proton cancer therapy system. Two years later, this program, which had been called "flagship theme," was ended [48]. Similarly, the Fox Chase program in Philadelphia was put on hold after four years [4].

In an interview in 2010, the Director of the Fox Chase Cancer Center called cyclotron accelerators "monsters" and further stated that a single cyclotron would take up an entire building and cost between 150 and 250 million USD [4]. But even in 2010, turn-key proton therapy facilities from various providers were on the market and none of these multiroom centers featured a cyclotron which was near the size or cost quoted. In fact, today's compact cyclotrons are smaller than a typical magnetic resonance imager.

The original idea of laser-driven medical accelerators should have been to study plasma-target interaction, develop new energy selection systems, or analyze unusual radiobiological effects that might result from ultrashort ion pulses. Once performance had been experimentally established, then suitable applications could have been pursued based on demonstrated performance. Instead, the chief thrust has been to replace a successful treatment methodology for cancer. This goal has not been met and will not come true at least in the next decade.

One could ask if there are no other meaningful applications for potential laser accelerators apart from proton therapy that are easier to achieve; or whether the term "cancer treatment" is such a successful buzzword that research funding is more likely than for projects that promise new insight into fundamental optical and plasma physics?

An important point to be kept in mind relates to the evolution of a new technology, in this case laser-based IBT, from laboratory to clinic. The size of today's petawatt lasers is extremely large, as were the first machines delivering
IBT, e.g. the Harvard Cyclotron and the Bevalac at Berkeley. However, the clinical value of the Bragg peak was demonstrated there, and led ultimately to the development of the clinically based facilities being installed today. The path was long: from the 1970s to 1990 when the first clinical proton therapy facility was opened in Loma Linda, USA, and another decade for the first commercially manufactured IBA installation. The fight for regulatory approval was also lengthy and complex, fought successfully first by Dr. James Slater at Loma Linda University. It is most likely that even if the required proton energy and intensity could be obtained from the very large research lasers, one can count on a several-decade development period before the technology will be ready and approved for the clinic.

\section{CONCLUSION}

Ten years after our first report, it is obvious that the idea of a laser-driven ion accelerator for clinical use is still many years from reality. Neither beam energy nor energy spread and beam intensity have reached the required level. Beam control, dose control, reproducibility of the pulses, reliability, durability, safety of the equipment, and last but not least, cost are still unresolved issues.

Laser-driven ion accelerators are in reality still a completely new high-risk technology concept. Model laser systems for ion acceleration continue to be large and expensive, whereas cyclotrons have indeed been reduced to tabletop size and are part of single-room proton beam facilities. The field of laser-induced acceleration still has to work on basic principles, while industry-based proton therapy with conventional accelerators is now fine-tuning the pencil-beam scanning dose delivery algorithms for even better flexibility and precision of conformation and normaltissue sparing. In addition, the market is blossoming as sizes and prices drop; today more than 60 facilities are treating patients, and many more are under construction.

Continuing research in laser-acceleration mechanisms and how to use these protons or ions for therapy might be an intriguing endeavor. However, claiming readiness for front-line applications while so many hurdles still remain will ultimately cause disillusionment due to unmet promises and will discredit the field. It will also harm the IBT industry since potential users might delay their decisions based on false hopes for smaller and cheaper solutions.

We welcome news of new achievements in laser performance and understanding of beam generating and control mechanisms, but urge temperance in making bold projections of performance beyond reasonable limits.

[1] U. Linz and J. Alonso, What does it take for laser driven proton accelerators to be applied to tumor therapy?, Phys. Rev. ST Accel. Beams 10, 094801 (2007). 
[2] S. V. Bulanov and V.S. Khoroshkov, Feasibility of using laser ion accelerators in proton therapy, Plasma Phys. Rep. 28, 453 (2002).

[3] V. Malka, S. Fritzler, E. Lefebvre, E. d'Humières et al. Practicability of protontherapy using compact laser systems, Med. Phys. 31, 1587 (2004).

[4] M. Martin, Laser accelerated radiotherapy: Is it on the way to the clinic?, J. Natl. Cancer Inst. 101, 450 (2009).

[5] K. M. Hofmann, S. Schell, and J. J. Wilkens, Laser-driven beam lines for delivering intensity modulated radiation therapy with particle beams, J. Biophoton. 5, 903 (2012).

[6] B. M. Hegelich, I. Pomerantz, L. Yin, H. C. Wu et al., Laser-driven ion acceleration from relativistically transparent nanotargets, New J. Phys. 15, 085015 (2013).

[7] K. P. Gall, The single-room ion beam facility, in Ion Beam Therapy-Fundamentals, Technology, Clinical Applications, edited by U. Linz (Springer-Verlag Berlin, 2012), pp. 661-72.

[8] R. Mayer and S. Vatnitsky, New facilities: plans and proposals, in Ion Beam Therapy-Fundamentals, Technology, Clinical Applications, edited by U. Linz (Ref. [7]), pp. 687-701.

[9] PTCOG statistics: http://www.ptcog.ch/archive/patient_ statistics/ijpt-15-00013.pdf, 2016.

[10] E. R. Dennis, M. R. Bussière, A. Niemierko, M. W. Lu et al., A comparison of critical structure dose and toxicity risks in patients with low grade gliomas treated with IMRT versus proton radiation therapy, Technol. Cancer Res. Treat. 12, 1 (2013).

[11] L. L. Lin, S. Vennarini, A. Dimofte, D. Ravanelli, K. Shillington, S. Batra, Z. Tochner, S. Both, and G. Freedman, Proton beam versus photon beam dose to the heart and left anterior descending artery for left-sided breast cancer, Acta Oncologica 54, 1032 (2015).

[12] R. Snavely, M. Key, S. Hatchett, T. Cowan et al., Intense High-Energy Proton Beams from Petawatt-Laser Irradiation of Solids, Phys. Rev. Lett. 85, 2945 (2000).

[13] S. A. Gaillard, T. Kluge, K. A. Flippo, M. Bussmann et al., Increased laser-accelerated proton energies via direct laser-light-pressure acceleration of electrons in microcone targets, Phys. Plasmas 18, 056710 (2011).

[14] B. M. Hegelich, D. Jung, B. J. Albright, M. Cheung et al., $160 \mathrm{MeV}$ laser-accelerated protons from $\mathrm{CH} 2$ nanotargets for proton cancer therapy, http://arxiv.org/abs/1310.8650, 2013.

[15] M. Borghesi and U. Schramm, Summary of Working Group 2: Ion beams from plasmas, Nucl. Instrum. Methods Phys. Res., Sect. A 829, 137 (2016).

[16] E. d'Humières, E. Lefebvre, L. Gremillet, and V. Malka, Proton acceleration mechanisms in high-intensity laser interaction with thin foils, Phys. Plasmas 12, 062704 (2005).

[17] J. Fuchs, P. Antici, E. d'Humières, E. Lefebvre et al., Laser-driven proton scaling laws and new paths towards energy increase, Nat. Phys. 2, 48 (2006).

[18] S. M. Pfotenhauer, O. Jäckel, A. Sachtleben, J. Polz et al., Spectral shaping of laser generated proton beams, New J. Phys. 10, 033034 (2008).

[19] K. Zeil, S. D. Kraft, S. Bock, M. Bussmann, T. E. Cowan, T. Kluge, J. Metzkes, T. Richter, R. Sauerbrey, and U. Schramm, The scaling of proton energies in ultrashort pulse laser plasma acceleration, New J. Phys. 12, 045015 (2010).

[20] D. Jung, L. Yin, B. J. Albright, D. C. Gautier et al., Efficient carbon ion beam generation from laser driven volume acceleration, New J. Phys. 15, 023007 (2013).

[21] C. M. Ma, R. L. Maughan, and C. Orton, Point/ counterpoint: Within the next decade conventional cyclotrons for proton therapy will become obsolete and replaced by far less expensive machines using compact laser systems for the acceleration of the protons, Med. Phys. 33, 571 (2006).

[22] K. W. D. Ledingham, P. R. Bolton, N. Shikazono, and C. M. C. Ma, Towards laser driven hadron cancer radiotherapy: A review of progress, Appl. Sci. 4, 402 (2014).

[23] B. Damato, Treatment of primary intraocular melanoma, Expert Rev. Anticancer Ther. 6, 493 (2006).

[24] H. Tsujii, personal communication, 2010.

[25] T. Bartal, M.E. Foord, C. Bellei, M. H. Key et al., Focusing of short-pulse high-intensity laser-accelerated proton beams, Nat. Phys. 8, 139 (2012).

[26] I. Hofmann, J. Meyer-ter-Vehn, X. Yan, A. Orzhekhovskaya, and S. Yaramyshev, Collection and focusing of laser accelerated ion beams for therapy applications, Phys. Rev. ST Accel. Beams 14, 031304 (2011).

[27] I. Hofmann, J. Meyer-ter-Vehn, X. Yan, and H. Al-Omari, Chromatic energy filter and characterization of laseraccelerated proton beams for particle therapy, Nucl. Instrum. Methods Phys. Res., Sect. A 681, 44 (2012).

[28] K. W. D. Ledingham, P. McKenna, T. McCanny, S. Shimizu et al. High power laser production of short-lived isotopes for positron emission tomography, J. Phys. D 37, 2341 (2004).

[29] E. Amato, A. Italiano, D. Margarone, B. Pagano, S. Baldari, and G. Korn, Study of the production yields of $18 \mathrm{~F}, 11 \mathrm{C}, 13 \mathrm{~N}$ and $15 \mathrm{O}$ positron emitters from plasmalaser proton sources at ELI-beam lines for labeling of PET radiopharmaceuticals, Nucl. Instrum. Methods Phys. Res., Sect. A 811, 1 (2016).

[30] C. A. J. Palmer, N. P. Dover, I. Pogorelsky, M. Babzien et al., Monoenergetic proton beams accelerated by a radiation pressure driven shock, Phys. Rev. Lett. 106, 014801 (2011).

[31] T. Z. Esirkepov, M. Yamagiwa, and T. Tajima, Laser ionacceleration scaling laws seen in multiparametric particlein-cell simulations, Phys. Rev. Lett. 96, 105001 (2006).

[32] D. Haberberger, S. Tochitsky, F. Fiuza, and C. Gong, R. A. Fonseca, L. O. Silva, W. B. Mori, and C. Joshi Collisionless shocks in laser-produced plasma generate monoenergetic high-energy proton beams, Nat. Phys. 8, 95 (2012).

[33] C. Obcemea, Potential clinical impact of laser-accelerated beams in cancer ion therapy, Nucl. Instrum. Methods Phys. Res., Sect. A 829, 149 (2016).

[34] H. Schwörer, S. Pfotenhauer, O. Jäckel, K. U. Amthor, B. Liesfeld, W. Ziegler, R. Sauerbrey, K. W. D. Ledingham, and T. Esirkepov, Laser plasma acceleration of quasimonoenergetic protons from microstructured targets, Nature (London) 439, 445 (2006).

[35] S. Schell and J. J. Wilkens, Advanced treatment planning methods for efficient radiation therapy with laser accelerated proton and ion beams, Med. Phys. 37, 5330 (2010). 
[36] G. A. P. Cirrone, M. Carpinelli, G. Cuttone, S. Gammino et al., ELIMED, future hadrontherapy applications of laseraccelerated beams, Nucl. Instrum. Methods Phys. Res., Sect. A 730, 174 (2013).

[37] F. Schillaci, A. Anzalone, G. A. P. Cirrone, M. Carpinelli et al., ELIMED, MEDical and multidisciplinary applications at ELI-beam lines, J. Phys. Conf. Ser. 508, 012010 (2014).

[38] C. L. S. Lewis, G. Nersisyan, M. Borghesi, D. Doria et al., The TARANIS laser: A multi-terawatt system for laser plasma physics. XXVII Internal Conference on Photonic, Electronic and Atomic Collisions (ICPEAC 2011), J. Phys. Conf. Ser. 388, 1044 (2012).

[39] O. Novak, M. Divoky, H. Turcicova, and P. Straka, Design of a petawatt optical parametric chirped pulse amplification upgrade of the kilojoule iodine laser PALS, Laser Part. Beams 31, 211 (2013).

[40] F. Schillaci, G. A. P. Cirrone, G. Korn, M. Maggiore et al., ELIMED: Medical application at ELI-beam lines. Status of the collaboration and first results, Acta Polytech. 54, 285 (2014).
[41] V. Derenchuk, The ProNova SC360 Gantry, in Workshop on Modern Hadron Therapy Gantry Developments (Cockcroft Institute, Daresbury, 2014), http://indico.hep .manchester.ac.uk/getFile.py/access?contribId=27\&resId= 0\&materialId=slides \& confId $=4226$.

[42] Y. Iwata, K. Noda, T. Shirai, T. Fujita et al., Present status of a superconducting rotating gantry for carbon therapy, in Proceedings of HIAT 2015, Yokohama, Japan, 2015, http:// www.rarf.riken.jp/hiat2015/proceedings/papers/frm1c01 .pdf.

[43] J. Fan, W. Luo, E. Fourkal, T. Lin, J. Li, I. Veltchev, and C.-M. Ma, Shielding design for a laser-accelerated proton therapy system, Phys. Med. Biol. 52, 3913 (2007).

[44] IBA:http://www.iba-protontherapy.com/proteusone-0, 2016.

[45] CNRS News 2006: http://www2.cnrs.fr/en/518.htm, 2016.

[46] V. Malka, Laser plasma accelerators for cancer treatment. Frontiers in Optics 2011/Laser Science XXVII, San Jose, CA, USA, 2011, OSA Technical Digest FThB2.

[47] Optics.org: Amplitude Systèmes agrees $€ 30 \mathrm{M}$ equity deal, 2012, http://optics.org/news/3/2/5, 2016.

[48] http://133.188.30.80/closed/pmrc-en.htm, 2016. 